\title{
USO DE CALCÁRIO AGRÍCOLA, GESSO AGRÍCOLA E CAL HIDRATADA AGRÍCOLA EM SISTEMA AGROPASTORIL NO NOROESTE PAULISTA: EFEITOS NA CULTURA DO MILHO
}

\author{
BORGES, Wander Luis Barbosa ${ }^{1}$; HIPÓLITO, Jorge Luiz ${ }^{2}$; BENSCH, Carlos Henrique ${ }^{3}$; TOKUDA, \\ Flávio $\mathrm{Sueo}^{4}$; SOUZA, Isabela Malaquias Dalto de ${ }^{5}$; FREITAS, Rogério Soares $\mathrm{de}^{1}$; \\ MATEUS, Gustavo Pavan ${ }^{6}$
}

ISSUE DOI: $10.3738 / 1982.2278 .3622$

\begin{abstract}
RESUMO: O presente trabalho teve por objetivo avaliar o efeito da aplicação de calcário agrícola, gesso agrícola e cal hidratada agrícola sobre as características agronômicas da cultura do milho, cultivado em sistema agropastoril, nas condições edafoclimáticas da região Noroeste Paulista. Os parâmetros avaliados na cultura do milho foram: altura de inserção da primeira espiga, altura de plantas, estande final $\mathrm{ha}^{-1}$, número de espigas ha ${ }^{-1}$, massa de cem grãos e produtividade de grãos. O delineamento experimental utilizado foi em blocos casualizados com sete tratamentos (T1: tratamento padrão: sem o uso de calcário agrícola, gesso agrícola e cal hidratada agrícola e sem revolvimento do solo; T2: calcário agrícola e gesso agrícola em superfície no primeiro ano e calcário agrícola em superfície no segundo ano; T3: calcário agrícola e gesso agrícola incorporados no primeiro ano e ambos em superfície no segundo ano; T4: calcário agrícola incorporado no primeiro ano e em superfície no segundo ano; T5: cal hidratada agrícola e gesso agrícola em superfície; T6: cal hidratada agrícola em sub superfície na dose de $450 \mathrm{~kg}$ $\mathrm{ha}^{-1}$ e gesso agrícola em superfície no primeiro ano e cal hidratada agrícola em superfície no segundo ano; T7: cal hidratada agrícola em sub superfície na dose de $260 \mathrm{~kg} \mathrm{ha}^{-1}$ e gesso agrícola em superfície no primeiro ano e cal hidratada agrícola em superfície no segundo ano) e quatro repetições. Os dados foram submetidos ao teste $\mathrm{F}$ e as médias foram comparadas pelo teste de Tukey $(\mathrm{p}<0,05)$. Constatou-se que a aplicação de cal hidratada agrícola na dose de $260 \mathrm{~kg} \mathrm{ha}^{-1}$ ao longo do perfil do solo, associado ao uso de gesso agrícola em superfície e a aplicação de cal hidratada agrícola em superfície no ano seguinte propiciou maior produtividade de grãos da cultura do milho e que o revolvimento do solo para incorporação de calcário agrícola e gesso agrícola no ano anterior comprometeu a produtividade de grãos da cultura do milho.
\end{abstract}

Palavras-chave: Zea mays (L.). Características agronômicas. Corretivos.

\section{AGRICULTURAL LIMESTONE, GYPSUM AND HYDRATED LIME USE IN AGROPASTORAL SYSTEM IN THE NORTHWEST OF SÃO PAULO STATE, BRAZIL: EFECTS ON MAIZE CROP}

\begin{abstract}
SUMMARY: The objective of this work was to evaluate the effect of the application of agricultural lime, agricultural gypsum and agricultural hydrated lime on the agronomic characteristics of the maize crop, cultivated in agropastoral system, under the edaphoclimatic conditions of the Northwest region of São Paulo State, Brazil. The parameters evaluated in the maize crop were: height of insertion of the first cob, height of plants, final stand ha ${ }^{-1}$, number of cobs ha ${ }^{-1}$, mass of one hundred grains and grain productivity. The experimental design was a randomized block with seven treatments (T1: standard treatment: without the use of agricultural limestone, agricultural gypsum and hydrated agricultural lime and no tillage; T2: agricultural limestone and agricultural gypsum on the surface in first year and agricultural limestone on the surface in second year; T3: agricultural limestone and agricultural gypsum incorporated in first year and both on the surface in second year; T4: agricultural limestone incorporated in first year and on the surface in second year; T5: agricultural hydrated lime and agricultural gypsum on the surface; T6: hydrated lime on the sub surface, at a dose of $450 \mathrm{~kg} \mathrm{ha}^{-1}$ and agricultural gypsum on the surface in first year and hydrated agricultural lime on the surface in second year; T7: hydrated agricultural lime on sub surface, at a dose of

\footnotetext{
${ }^{1}$ Pesquisador Científico, Dr. - IAC - CAP Seringueira e Sistemas Agroflorestais, Votuporanga, SP;

${ }^{2}$ Assistente Agropecuário - CDRS - DSMM/NPS Araçatuba, Araçatuba, SP;

${ }^{3}$ Assistente Agropecuário - CDRS - CA Brejo Alegre, Brejo Alegre, SP;

${ }^{4}$ Assistente Agropecuário - CDRS - CA Riolândia, Riolândia, SP;

${ }^{5}$ Doutoranda, MSc. - UNESP - Faculdade de Engenharia, Ilha Solteira, SP;

${ }^{6}$ Pesquisador Científico, Dr. - APTA - PRDTA Extremo Oeste, Andradina, SP.
} 
$260 \mathrm{~kg} \mathrm{ha}^{-1}$ and agricultural gypsum on the surface in first year and hydrated agricultural lime on the surface in second year) and four replications. The data were submitted to the F test and the means were compared by the Tukey test ( $\mathrm{p}<0.05)$. It was verified that the application of agricultural hydrated lime at a dose of $260 \mathrm{~kg} \mathrm{ha}^{-1}$ along the soil profile, associated to the use of agricultural gypsum on the surface and the application of hydrated agricultural lime on the surface in the following year resulted in a higher corn grain productivity and that soil tillage for the incorporation of agricultural limestone and agricultural gypsum in the previous year compromised the grain productivity of the maize crop.

Keywords: Glycine max (L.) Merrill. Agronomic characteristics. Correctives.

\section{INTRODUÇÃO}

Com o aumento da demanda por alimentos e a evolução tecnológica na produção, a atividade agrícola moderna passou a se caracterizar por sistemas padronizados e simplificados de monocultura, os quais predominam nas propriedades rurais em todo o mundo, mas que têm apresentado sinais de saturação, em virtude da elevada demanda por energia e por recursos naturais que os caracterizam (BALBINO et al., 2011).

Uma opção interessante é a utilização de sistemas mistos de produção agrícola, os quais são mais sustentáveis que os sistemas especializados de produção de grãos e fibra (WILKINS, 2008), como os sistemas agropastoris manejados sob sistema de semeadura direta, que têm mostrado maior rentabilidade por área, maior diversificação de atividades, menor risco econômico e menor custo de produção (BALBINOT JÚNIOR et al., 2009; MACEDO, 2009), no entanto, o sucesso de um sistema consorciado depende do correto manejo das espécies e dos fatores de produção que afetam as espécies, visando a garantir retornos econômicos e ambientais satisfatórios (MELOTTO et al., 2009).

Nestes sistemas de produção, geralmente, a correção da acidez do solo é realizada mediante aplicação de calcário na superfície, sem incorporação e, a baixa mobilidade dos produtos de dissolução do calcário aplicado na superfície limita sua eficiência na redução da acidez em camadas sub superficiais de solos com cargas variáveis e, que dependem da lixiviação de sais, orgânicos e, ou, inorgânicos, através do perfil do solo (CAIRES et al., 2006).

Uma opção interessante para melhoria das camadas sub superficiais nestes sistemas é a utilização do gesso agrícola, o qual tem sido utilizado em solos ácidos como um produto complementar ao calcário (BRAGA; VALE; MUNIZ, 1995; SILVA et al., 1998) e, para se ajustar o pH dos solos e proporcionar melhores condições de crescimento radicular e, assim, melhorar o rendimento das culturas, podem ser utilizados o carbonato de cálcio, a cal virgem e a cal hidratada (EULA, 2018)

O presente trabalho teve por objetivo avaliar o efeito da aplicação de calcário agrícola, gesso agrícola e cal hidratada agrícola sobre as características agronômicas da cultura do milho, cultivada em sistema agropastoril, nas condições edafoclimáticas da região Noroeste Paulista.

\section{MATERIAL E MÉTODO}

O experimento foi instalado no mês de maio de 2009 no Centro Avançado de Pesquisa de Seringueira e Sistemas Agroflorestais, do Instituto Agronômico (IAC), da Agência Paulista de Tecnologia dos Agronegócios - APTA/SAA, localizado no município de Votuporanga, SP, (20²0'S, 4958'W e $510 \mathrm{~m}$ de altitude), em um Argissolo Vermelho-Amarelo eutrófico (segundo o SiBCS, SANTOS et al., 2013).

O clima é o tropical com invernos secos (Aw na classificação de Köppen) com temperatura média anual de $24^{\circ} \mathrm{C}$, tendo a média das máximas de $31,2^{\circ} \mathrm{C}$ e a média das mínimas de $17,4^{\circ} \mathrm{C}$. A precipitação pluviométrica média anual é de 1328,6 mm. 
O delineamento experimental utilizado foi o em blocos casualizados com quatro repetições, utilizando-se sete tratamentos:

T1 - Tratamento padrão, sem o uso de calcário agrícola, gesso agrícola e cal hidratada agrícola e sem revolvimento do solo;

T2 - $1^{\circ}$ Ano: aplicação em superfície de calcário agrícola, na dose de $2000 \mathrm{~kg} \mathrm{ha}^{-1}$ e de gesso agrícola, na dose de $2500 \mathrm{~kg} \mathrm{ha}^{-1} ; 2^{\circ}$ ano: aplicação, em superfície, de calcário agrícola para estabelecer que, o cálcio ocupe $65 \%$ da capacidade de troca de cátions (CTC) na camada de 0-0,20 m;

T3 - $1^{\circ}$ Ano: Aplicação de calcário agrícola, na dose de $1000 \mathrm{~kg} \mathrm{ha}^{-1}$, incorporado com gradagem aradora Rome e inversão da leiva com arado de aiveca + aplicação de calcário agrícola, na dose de 1350 $\mathrm{kg} \mathrm{ha}^{-1}$ e de gesso agrícola, na dose de $1500 \mathrm{~kg} \mathrm{ha}^{-1}$, incorporados com gradagem niveladora; $2^{\circ}$ ano: aplicação de calcário agrícola e gesso agrícola, em superfície, para estabelecer que, respectivamente, o cálcio ocupe $65 \%$ da CTC na camada de $0-0,20 \mathrm{~m}$ e $50 \%$ da capacidade de troca de cátions efetiva (CTCe) na camada de 0,20-0,40 m;

T4 - Aplicação de calcário agrícola, na dose de $1000 \mathrm{~kg} \mathrm{ha}^{-1}$, incorporado com gradagem aradora Rome e inversão da leiva com arado de aiveca + aplicação de calcário agrícola, na dose de $1350 \mathrm{~kg} \mathrm{ha}^{-1}$, incorporado com gradagem niveladora; $2^{\circ}$ ano: aplicação, em superfície, de calcário agrícola para estabelecer que, o cálcio ocupe $65 \%$ da CTC na camada de 0-0,20 m;

T5 - $1^{\circ}$ Ano: Aplicação em superfície de cal hidratada agrícola, na dose de $725 \mathrm{~kg} \mathrm{ha}^{-1}$ e de gesso agrícola, na dose de $2500 \mathrm{~kg} \mathrm{ha}^{-1} ; 2^{\circ}$ ano: Aplicação, em superfície, de cal hidratada agrícola e gesso agrícola para estabelecer que, respectivamente, o cálcio ocupe $65 \%$ da CTC na camada de $0-0,20$ m e 50\% da CTCe na camada de 0,20-0,40 m;

T6 - $1^{\circ}$ Ano: Aplicação em sub superfície de cal hidratada agrícola, na dose de $450 \mathrm{~kg} \mathrm{ha}^{-1} \mathrm{e}$ aplicação em superfície de gesso agrícola, na dose de $2500 \mathrm{~kg} \mathrm{ha}^{-1} ; 2^{\circ}$ ano: aplicação, em superfície, de cal hidratada agrícola para estabelecer que, o cálcio ocupe 65\% da CTC na camada de 0-0,20 m;

T7 - $1^{\circ}$ Ano: Aplicação em sub superfície de cal hidratada agrícola, na dose de $260 \mathrm{~kg} \mathrm{ha}^{-1} \mathrm{e}$ aplicação em superfície de gesso agrícola, na dose de $2500 \mathrm{~kg} \mathrm{ha}^{-1} ; 2^{\circ}$ ano: aplicação, em superfície, de cal hidratada agrícola para estabelecer que, o cálcio ocupe $65 \%$ da CTC na camada de 0-0,20 m.

A cal hidratada agrícola utilizada continha $40 \%$ de $\mathrm{CaO}$ e $27 \%$ de $\mathrm{MgO}$. O calcário agrícola utilizado continha $42 \%$ de $\mathrm{CaO}$ e $7 \%$ de $\mathrm{MgO}$. O gesso agrícola utilizado continha $17 \%$ de $\mathrm{Ca}$ e $14 \%$ de S.

A área era destinada à produção de grãos e sementes em sistema convencional de preparo de solo. Na safra 2014/15 foi cultivada mamona para produção de sementes. Na safra 2015/16 foi cultivado milho na primeira safra e Crotalaria juncea na segunda safra. Na safra 2016/17 foi cultivado milho em consórcio com Urochloa ruziziensis na primeira safra, em sistema de semeadura direta sobre a palhada da $C$. juncea. Na safra 2017/18 foi cultivada soja na primeira safra e C. juncea na segunda safra.

Em outubro de 2018 foi realizada uma coleta de amostras de solo para caracterização química (RAIJ et al., 2001) nas camadas de 0-0,10, 0,10-0,20, 0,20-0,40 e 0,40-0,80 m de profundidade, e os resultados estão apresentados na Tabela 1. 
Tabela 1. Caracterização química do solo, nas camadas de $0-0,10,0,10-0,20,0,20-0,40$ e 0,40-0,80 m, 2018 .

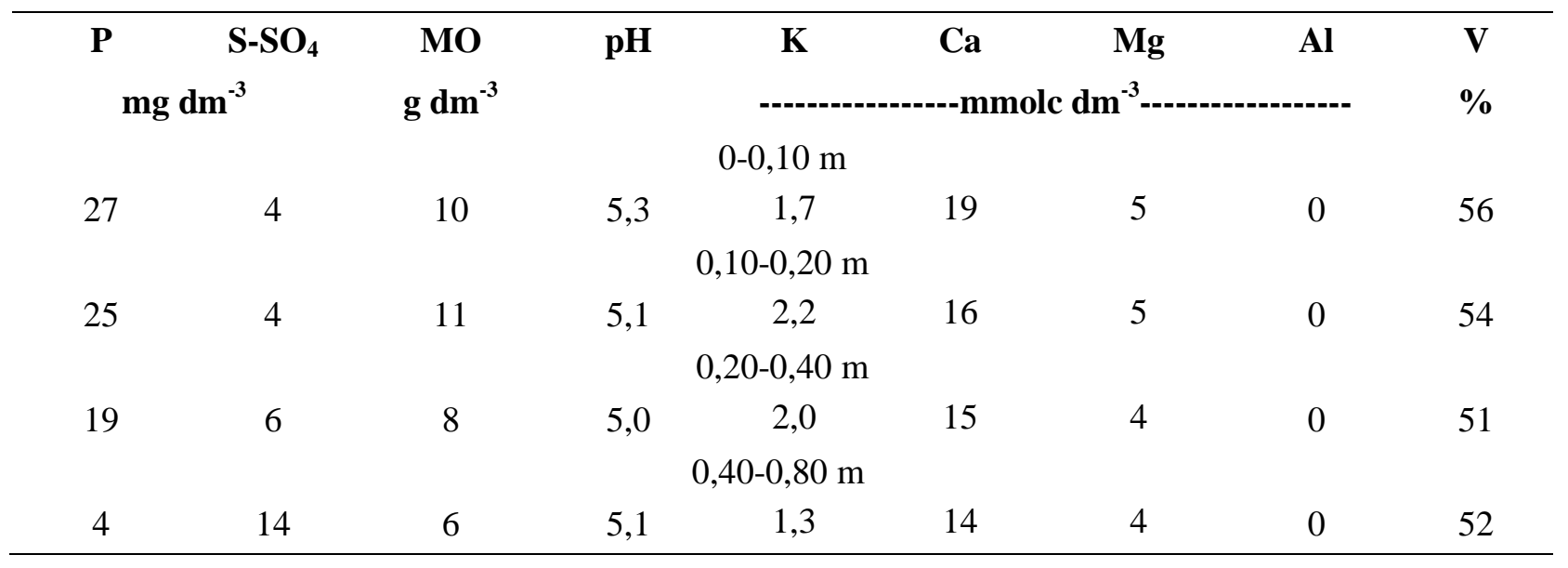

Foi realizada uma dessecação pré-plantio no dia 22/10/2018, utilizando-se glifosato $720 \mathrm{~g} \mathrm{~kg}^{-1}$, na dose de 2,0 $\mathrm{kg} \mathrm{ha}^{-1}$ do produto comercial (p.c.) + 2,4-diclorofenoxiacético $670 \mathrm{~g} \mathrm{~L}^{-1}$ na dose de $1,0 \mathrm{~L} \mathrm{ha}^{-1}$ do p.c. + óleo mineral, na dose de $1,0 \mathrm{~L} \mathrm{ha}^{-1}$ do p.c.

No dia 17/10/2018 realizou-se uma amostragem de quantidade de palhada presente na área. Foram retiradas duas amostras de $0,5 \times 0,5 \mathrm{~m}$ por parcela, as quais foram acondicionadas em sacos de papel e levadas para secagem em estufa de ventilação forçada, regulada a $65-70^{\circ} \mathrm{C}$ por 72 horas. A quantidade de matéria seca presente na área foi de $2424 \mathrm{~kg} \mathrm{ha}^{-1}$.

No dia 13/11/2018 foi realizada a aplicação do calcário em superfície nos tratamentos T2, T3 e T4.

No dia 14/11/2018 foi realizada a aplicação da cal hidratada agrícola em superfície nos tratamentos T5, T6 e T7.

No dia 26/11/2018 foi realizada a aplicação de gesso agrícola em superfície nos tratamentos T3 e T5.

A semeadura do milho foi realizada mecanicamente no sistema de semeadura direta sobre a palhada da $C$. juncea no dia 26/11/2018, utilizando-se o híbrido AG 8088 PRO2 no espaçamento de $0,8 \mathrm{~m}$ e população de 56250 plantas ha ${ }^{-1}$, com adubação de base na dose de $300 \mathrm{~kg} \mathrm{ha}^{-1}$ do adubo formulado 08$28-16$, com $1,7 \%$ de $\mathrm{Ca}$ e $3,6 \%$ de $\mathrm{S}\left(\mathrm{S}-\mathrm{SO}_{4}\right)$.

A semente foi tratada industrialmente com imidacloprido $150 \mathrm{~g} \mathrm{~L}^{-1}$ e tiodicarbe $450 \mathrm{~g} \mathrm{~L}^{-1}$, na dose de $0,3 \mathrm{~L} \mathrm{ha}^{-1}$.

Foi realizada uma aplicação glifosato $720 \mathrm{~g} \mathrm{~kg}^{-1}$, na dose de $2,5 \mathrm{~kg} \mathrm{ha}^{-1}$ do produto comercial (p.c.) + atrazina $500 \mathrm{~g} \mathrm{~L}^{-1}$ na dose de $3,0 \mathrm{~L} \mathrm{ha}^{-1}$ do p.c. para o controle de plantas daninhas, no dia $06 / 12 / 2018$.

Os parâmetros avaliados na cultura do milho foram: altura de inserção da primeira espiga, altura de plantas, estande final $\mathrm{ha}^{-1}$, número de espigas $\mathrm{ha}^{-1}$, massa de cem grãos e produtividade de grãos. As avaliações foram realizadas no momento da colheita do milho, realizada no dia 17/04/2019. A massa de cem grãos e a produtividade de grãos foi obtida padronizando-se a umidade dos grãos para 13\% (base úmida).

A amostragem da altura de inserção da primeira espiga e altura de plantas foi realizada em cinco plantas de cada parcela, e a amostragem do estande final ha ${ }^{-1}$, número de espigas ha ${ }^{-1}$, massa de cem grãos e produtividade de grãos foi realizada em 2 linhas de $5 \mathrm{~m}$ de cada parcela. 
As espigas foram debulhadas em debulhadora mecânica. Após a debulha os grãos foram pesados e mensurada sua umidade para o cálculo da produtividade de grãos. Em seguida separou-se 100 grãos para obtenção da massa de 100 grãos.

Os dados foram submetidos ao teste $\mathrm{F}$ e as médias foram comparadas pelo teste de Tukey ( $\mathrm{p}<0,05$ ), com o uso do programa computacional Assistat (SILVA; AZEVEDO, 2016).

Os dados mensais de evapotranspiração potencial, precipitação pluvial e temperatura média de Votuporanga, SP, no período de novembro de 2018 a abril de 2019, encontram-se na Figura 1.

Figura 1. Dados de evapotranspiração potencial (ETP), precipitação pluvial (PP) e temperatura média (T), em Votuporanga, SP, no período estudado, novembro de 2018 a abril de 2019. Fonte: CIIAGRO (2019).

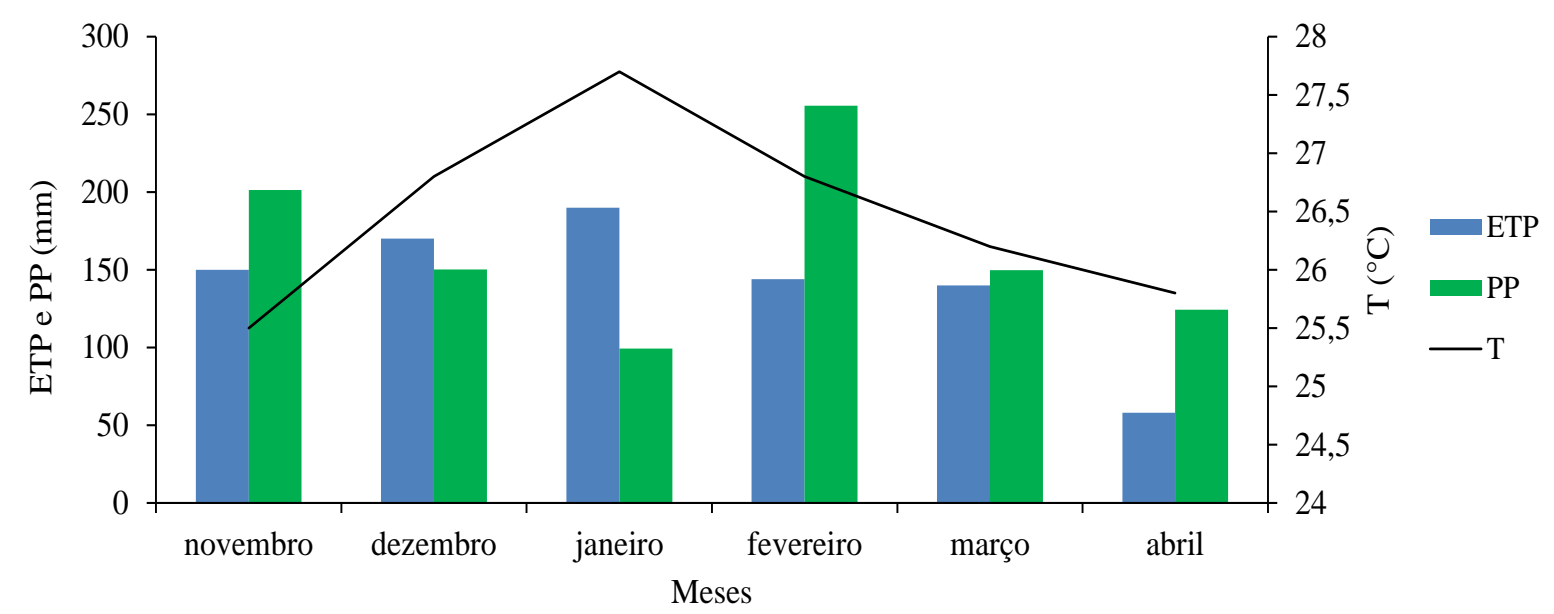

\section{RESULTADO E DISCUSSÃO}

As características agronômicas da cultura do milho estão demonstradas na Tabela 3. O tratamento T3 (calcário agrícola e gesso agrícola incorporados no primeiro ano e ambos em superfície no segundo ano) proporcionou menor produtividade de grãos e diferiu $(\mathrm{p}<0,05)$ do tratamento $\mathrm{T} 7$ (cal hidratada agrícola em sub superfície na dose de $260 \mathrm{~kg} \mathrm{ha}^{-1}$ e gesso agrícola em superfície no primeiro ano e cal hidratada agrícola em superfície no segundo ano).

Tabela 3. Características agronômicas da cultura do milho, Votuporanga, SP, 2019.

(Continua)

\begin{tabular}{|c|c|c|c|c|c|c|}
\hline Tratamentos $^{(1)}$ & $\begin{array}{r}\text { Altura de } \\
\text { inserção }^{(4)} \\
(\mathrm{n}\end{array}$ & $\begin{array}{l}\text { Altura de } \\
\text { plantas }\end{array}$ & Estande final & $\begin{array}{l}\text { Número de } \\
\text { espigas }\end{array}$ & $\begin{array}{l}\text { Massa de } \\
\text { cem grãos } \\
\text { (g) }\end{array}$ & $\begin{array}{c}\text { Produtividade } \\
\text { de grãos } \\
\left(\mathrm{kg} \mathrm{ha}^{-1}\right)\end{array}$ \\
\hline Padrão & $0,65^{(\mathrm{ns})}$ & 1,53 & 58750 & 58750 & 25,15 & $4798 \mathrm{ab}^{(5)}$ \\
\hline $\mathrm{T} 2$ & 0,62 & 1,53 & 53125 & 56667 & 25,55 & $4991 \mathrm{ab}$ \\
\hline $\mathrm{T} 3$ & 0,60 & 1,40 & 56563 & 57813 & 23,96 & $3781 \mathrm{~b}$ \\
\hline $\mathrm{T} 4$ & 0,58 & 1,45 & 56875 & 55313 & 25,25 & $4889 \mathrm{ab}$ \\
\hline $\mathrm{T} 5$ & 0,63 & 1,54 & 61250 & 58333 & 23,58 & $4913 \mathrm{ab}$ \\
\hline
\end{tabular}


Tabela 3. Características agronômicas da cultura do milho, Votuporanga, SP, 2019.

(Conclusão)

\begin{tabular}{|c|c|c|c|c|c|c|}
\hline Tratamentos $^{(1)}$ & $\begin{array}{r}\text { Altura de } \\
\text { inserção }^{(4)} \\
(n\end{array}$ & $\begin{array}{l}\text { Altura de } \\
\text { plantas }\end{array}$ & Estande final & $\begin{array}{l}\text { Número de } \\
\text { espigas }\end{array}$ & $\begin{array}{c}\text { Massa de } \\
\text { cem grãos } \\
\text { (g) }\end{array}$ & $\begin{array}{c}\text { Produtividade } \\
\text { de grãos } \\
\left(\mathrm{kg} \mathrm{ha}^{-1}\right)\end{array}$ \\
\hline T6 & 0,63 & 1,51 & 54375 & 55313 & 26,35 & $4838 \mathrm{ab}$ \\
\hline $\mathrm{T} 7$ & 0,63 & 1,56 & 57500 & 57500 & 25,42 & 5259 a \\
\hline $\mathrm{DMS}^{(2)}$ & 0,10 & 0,18 & 8253 & 8318 & 3,90 & 1427 \\
\hline $\mathrm{CV}^{(3)}$ & 6,95 & 5,13 & 6,21 & 6,24 & 6,67 & 12,78 \\
\hline
\end{tabular}

${ }^{(1)}$ Tratamento padrão: sem o uso de calcário agrícola, gesso agrícola e cal hidratada agrícola e sem revolvimento do solo, T2: calcário agrícola e gesso agrícola em superfície no primeiro ano e calcário agrícola em superfície no segundo ano, T3: calcário agrícola e gesso agrícola incorporados no primeiro ano e ambos em superfície no segundo ano, T4: calcário agrícola incorporado no primeiro ano e em superfície no segundo ano, T5: cal hidratada agrícola e gesso agrícola em superfície, T6: cal hidratada agrícola em sub superfície na dose de $450 \mathrm{~kg} \mathrm{ha}^{-1}$ e gesso agrícola em superfície no primeiro ano e cal hidratada agrícola em superfície no segundo ano, T7: cal hidratada agrícola em sub superfície na dose de $260 \mathrm{~kg} \mathrm{ha}^{-1}$ e gesso agrícola em superfície no primeiro ano e cal hidratada agrícola em superfície no segundo ano; ${ }^{(2)}$ DMS: Diferença mínima significativa; ${ }^{(3)} \mathrm{CV}$ : Coeficiente de variação; ${ }^{(4)}$ Altura de inserção: altura de inserção da primeira vagem; ${ }^{(5)}$ significativo a $5 \%$ de probabilidade; ${ }^{(n)}$ : não-significativo.

Enfatiza-se que o tratamento T3, em 2018, recebeu preparo convencional do solo com gradagem aradora e niveladora e aração, para incorporação do calcário agrícola, no perfil de 0-0,20 e 0,20-0,40 m, e do gesso agrícola, e o solo do local tem estrutura "extremamente frágil", pois apresenta apenas 10,0,11,8 e $14,2 \%$ de argila nas camadas de 0-0,20, 0,20-0,40 e 0,40-0,80 m, respectivamente.

Por outro lado, no tratamento T7, em 2018, foi realizada a aplicação da cal hidratada agrícola até a camada de $0,57 \mathrm{~m}$, através de escarificação, a qual provavelmente rompeu uma possível camada adensada em sub superfície presente na área, considerando que a mesma foi cultivada em sistema convencional de preparo do solo até a safra 2015/16, o que pode ter levado à formação de "pé-de-arado ou pé-de-grade".

O rompimento desta possível camada adensada e o fornecimento de $\mathrm{Ca}$, nutriente com papel preponderante no crescimento radicular das plantas (RITCHEY et al., 1982), até a camada de 0,57 m, no primeiro ano e, em superfície no segundo ano, pode ter favorecido o desenvolvimento do sistema radicular da cultura do milho e, consequentemente, melhorado a absorção de água e nutrientes, reduzindo os efeitos do déficit hídrico pronunciado ocorrido no pleno florescimento da cultura do milho, em janeiro de 2018, devido à alta evapotranspiração, baixa pluviosidade e altas temperaturas médias (Figura 1). Segundo Santana, Landau e Sans (2018), por ocasião da floração, temperaturas médias superiores a $26^{\circ} \mathrm{C}$ aceleram o desenvolvimento dessa fase, e inferiores a $15,5^{\circ} \mathrm{C}$ o retardam e, cada grau acima da temperatura média de $21,1^{\circ} \mathrm{C}$, nos primeiros sessenta dias após a semeadura, pode acelerar o florescimento em dois a três dias, e temperaturas noturnas superiores $24^{\circ} \mathrm{C}$, proporcionam um aumento da respiração, ocasionando uma diminuição da taxa de redistribuição de fotoassimilados e consequente redução da produtividade.

\section{CONCLUSÃO}

A aplicação de cal hidratada agrícola na dose de $260 \mathrm{~kg} \mathrm{ha}^{-1}$ ao longo do perfil do solo, associado ao uso de gesso agrícola em superfície e a aplicação de cal hidratada agrícola em superfície no ano seguinte propiciou maior produtividade de grãos da cultura do milho em sistema agropastoril no Noroeste Paulista. 
O revolvimento do solo para incorporação de calcário agrícola e gesso agrícola no ano anterior comprometeu a produtividade de grãos da cultura do milho em sistema agropastoril no Noroeste Paulista.

\section{AGRADECIMENTOS}

À Votorantim Cimentos e Agronelli Indústria e Comércio de Insumos Agropecuários Ltda pelo apoio financeiro ao projeto que originou este artigo.

\section{REFERÊNCIAS}

BALBINO, L. C.et al. Evolução tecnológica e arranjos produtivos de sistemas de integração lavourapecuária floresta no Brasil. Pesquisa Agropecuária Brasileira. v. 46, n. 10, p. i-xii, 2011. Disponível em: http://dx.doi.org/10.1590/S0100-204X2011001000001

BALBINOT JÚNIOR, A. A.et al. Integração lavoura-pecuária: intensificação de uso de áreas agrícolas. Ciência Rural, v. 39, n. 6, p. 1925-1933, 2009. Disponível em:http://dx.doi.org/10.1590/S0103$\underline{84782009005000107}$

BRAGA, F. A.; VALE, F. R.; MUNIZ, J. A. Movimentação de nutrientes no solo, crescimento e nutrição mineral no eucalipto, em função de doses de gesso e níveis de irrigação. Revista Brasileira de Ciência do Solo, v. 19, p. 69-77, 1995.

CAIRES, E. F.; BARTH, G.; GARBUIO, F. J. Lime application in the establishment of a no-till system for grain crop production in Southern Brazil. Soil and Tillage Research, v. 89, n. 1, p. 3-12, 2006. Disponível em:https://doi.org/10.1016/j.still.2005.06.006

CENTRO INTEGRADO DE INFORMAÇÕES AGROMETEOROLÓGICAS - CIIAGRO. Resenha: Votuporanga no período de 01/11/2018 até 30/04/2019. São Paulo, 2019. Disponível em: http://www.ciiagro.sp.gov.br/ciiagroonline/Listagens/Resenha/LResenhaLocal.asp

SANTOS, H. G.et al. Centro Nacional de Pesquisa de Solos. Sistema brasileiro de classificação de solos. 3. ed. Rio de Janeiro, 2013. 353 p.

EUROPEAN LIME ASSOCIATION AISBL - EULA. Lime applications: agriculture, forestry and fish farming. 2018. Disponível em:https://www.eula.eu/agriculture-forestry-and-fish-farming

MACEDO M. C. M. Integração lavoura e pecuária: o estado da arte e inovação tecnológicas. Revista Brasileira de Zootecnia, v. 38, p. 133-46, 2009. Disponível em: http://www.scielo.br/pdf/rbz/v38nspe/v38nspea15.pdf

MELOTTO, A.et al. Sobrevivência e crescimento inicial em campo de espécies florestais nativas do Brasil Central indicadas para sistemas silvipastoris. Revista Árvore, v. 33, n. 3, p. 425-432, 2009. Disponível em:http://dx.doi.org/10.1590/S0100-67622009000300004

RAIJ, B. van.et al. Análise química para avaliação da fertilidade do solo. Campinas: Instituto Agronômico, 2001.

RITCHEY, K. D.; SILVA, S. E.; COSTA, V. F. No TitCalcium deficiency in clayey B horizons of savannah Oxisolsle. Soil Science, v. 133, p. 378-382, 1982. 
SANTANA, D. P; LANDAU, E. C.; SANS, L. M. A. Clima e solo. In: Cultivo do milho. Sistemas de Produção, 1. Embrapa Milho e Sorgo. Disponível em:

https://www.spo.cnptia.embrapa.br/conteudo?p_p id=conteudoportlet WAR sistemasdeproducaolf6 1ga 1 ceportlet\&p_p_lifecycle $=0 \& p \_p \_s t a t e=$ normal\&p_p_mode $=$ view $\&$ p_p_col_id $=$ column-

$2 \& p$ p col pos $=1 \& p$ p col count $=2 \& p$ r $\_$p -76293187 sistemaProducaoId $=3821 \& p$ r $\_$p 996514994_topicoId $=3718$

SILVA, F. A. S.; AZEVEDO, C. A. V. The Assistat Software Version 7.7 and its use in the analysis of experimental data. African Journal of Agricultural Research, v. 11, n. 39, p. 3733-3740, 2016. Disponível em: http://www.academicjournals.org/journal/AJAR/article-abstract/5E8596460818

SILVA, A. A.et al. Efeitos de relações $\mathrm{CaSO}_{4} / \mathrm{CaCO}^{3}$ na mobilidade de nutrientes no solo e no crescimento. Revista Brasileira de Ciência do Solo, v. 22, n. 3, p. 451-457, 1998. Disponível em: http://dx.doi.org/10.1590/S0100-06831998000300011

WILKINS, R. J. Eco-efficient approaches to land management: a case for increased integration of crop and animal production systems. Philosophical Transactions of the Royal Society B - Biological Sciences, v. 363, n. 1491, p. 517-525, 2008. Disponível em: http://rstb.royalsocietypublishing.org/content/363/1491/517 